\title{
Quantum-Level Phenomena in Nanowires
}

\author{
N. Garcia, J. L. Costa-Krämer \\ Laboratorio de Física de Sistemas Pequeños (CSIC-UAM), Universidad Autónoma de Madrid
}

\begin{abstract}
Table-top experiments, in which very small filaments are formed during the vibrationinduced breaking of the contact between a pair of wires, can be used to study quantum-leve effects at room temperature, such as quantized electrical conductance and non-linearity at high excitations leading to black-body light emission.
\end{abstract}

Technologically important quantum effects revealed by step-like changes in conductance are normally observed and applied by fabricating extremely small devices. These fundamental effects arise when conduction takes place in a conductor with a cross-section of the order of the wavelength of the charge carriers involved. When the conductor's length is less than the inelastic mean free path of the carrier, scattering takes place mainly at the boundaries. Conduction will be ballistic, that is, independent of materials properties and determined only by the geometry and the electron density, if defects or surface roughness that produce elastic scattering momentum transfer, but no energy transfer - are absent. Quantization of electrical conductance in the ballistic regime, revealed by oscillatory or staircase behaviour in current-voltage curves staircase, is possible if the electrons are confined by boundaries on a length scale of the order of the Fermi wavelength of the conducting electrons.

A simple calculation shows that the width of a metallic conductor cannot be larger than 2-3 nm for quantum effects to be observed at room temperature. This is because the quantized electronic states involved in the conductance have to be separated by an energy of at least $0.1 \mathrm{eV}$ (300 K corresponds to approximately $0.026 \mathrm{eV})$. One approach for obtaining widths of the order of the Fermi wavelength $\lambda$ exploits metallic nanowires.

Nicolas Garcia is the Director of the Laboratorio de Física de Sistemas Pequeños (CSIC-UAM), Facultad de Ciencas, Universidad Autónoma de Madrid, Cantoblanco, E-28049 Madrid. He studied physics in Madrid and received a PhD from the Universidad Autónoma in 1975. Professor Garcia joined the university's academic staff in 1978 and he has worked as a visting scientist in several laboratories in the USA. Jose Luis Costa-Krämer is a postdoc with the Laboratorio de Fisica de Sistemas Pequeños (CSIC-UAM). He studied physics in Madrid and received his PhD from the Royal Institute of Technology, Stockholm, in 1994.
Binnig and Rohrer's invention in 1981 of the scanning tunnelling microscope (STM) opened up a new way for understanding physics and the world at the nanoscale. The STM is able to keep a stable current at a vacuum gap of about $1 \mathrm{~nm}$ between tip and sample. Imaging metallic surfaces with nanometre resolution by scanning the tip while monitoring various modes of current feedback is common practice nowadays. The STM has also led to the development of local probes that employ other interactions to image surfaces. Finally, using STM techniques one can touch an object and study the properties of a nanocontact.

A theoretical prediction that the conductance observed when a STM tip touched a sample should exhibit staircase behaviour at room temperature as a function of the conductor width was presented in 1987 [1]. Some experimental results hinted at support for this prediction [2]. The idea was simple: when a metallic tip was pushed into a metallic sample the section of the contact should change, and consequently the conductance for a fixed applied voltage $V$. At the first stages of the contact, the contact would be on the nanometre size and, with $\lambda$ of the order of 0.5 $\mathrm{nm}$, the conductance should exhibit oscillatory or staircase behaviour. Two papers presented in 1988 reported experimental evidence for this phenomenon in a GaAs constriction at $0.6 \mathrm{~K}$, where low temperatures were necessary because the electron wavelength was about $30 \mathrm{~nm} \mathrm{[3].}$

The first reports of conductance quantization at room temperature measured using a STM appeared in 1993 and 1994 [4, 5]. The experiment consisted in pushing a STM tip into a metal and then pulling it back. Numerical simulations [6] predicted that a nanowire formed between the metallic sample and the tip when the tip was retracted. As the tip was pulled fur- ther back, the nanowire gradually decreased in cross-section, with the conductance behaving like a staircase function for a constant applied voltage.

A more general and striking observation was made in 1995: in order to detect conductance quantization at room temperature it was unnecessary to crush a STM tip into a sample. Driving two macroscopic wires in and out of contact demonstrated a much more clearly resolved staircase conductance. It was proposed that the contact between the macroscopic metals did not fracture cleanly, but was stretched into many filaments, in much the same way as what happens to the mozarella cheese when a pizza is broken. Quantized conductance was observed in a single nanofilament at the last stages of contact breaking, when the filament that remained had the best nanomechanical properties and left behind broken filaments that could not stretch to the limit [7].

The experiment was very simple to perform and represented a beautiful observation of a quantum mechanical effect. A potential difference was maintained between two household wires fixed

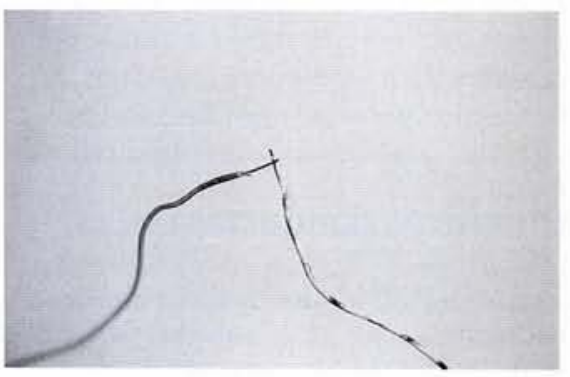

Fig. 1. A photograph of the ends of a pair of loosely touching copper wires, $0.6 \mathrm{~mm}$ in diameter, which were used in the table-top quantum conductance experiments. There was a potential difference of a few tens of $\mathrm{mV}$ between the wires and their other ends were connected to an oscilloscope via an $I-V$ converter having a gain of $10^{5}$.

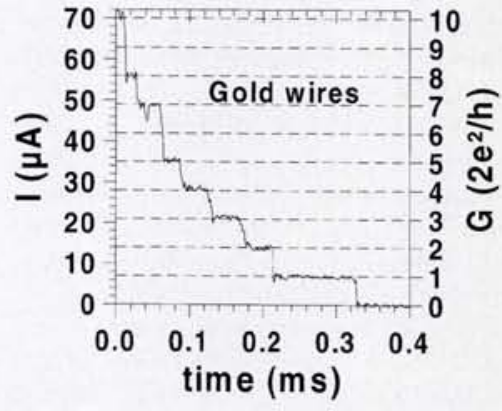

Fig. 2. The conductance at the final stages of the fracture of a macroscopic gold contact formed between two high-purity gold wires in air at room temperature. The applied potential difference is $90.35 \mathrm{mV}$, which corresponds to quantum steps of $7 \mu \mathrm{A}\left(2 e^{2} / h\right.$ is $\left.77.5 \mu \mathrm{S}\right)$. 
to a table with their ends lightly touching (Fig. 1). The table was then tapped and as the wires vibrated the contact between them broke to reveal staircase traces of the current on an oscilloscope.

Fig. 2 gives a characteristic trace displaying conductance quantization. It was obtained by plotting the current as a function of time at the point when the contact between high-purity gold wires in air at room temperature was in the final stages of breaking. Experiments demonstrating quantized conductance using macroscopic wires have also been performed with $\mathrm{Cu}$, Pt and amorphous metals in an ultra-high vacuum and submerged in oil, glycerin and water [7]. We have also been studied liquid metals ( $\mathrm{Hg}$ at room temperature and $\mathrm{Sn}$ at $300 \mathrm{~K})$. However, it must be appreciated that other curves did not reveal such clear evidence for quantization since the conductance plateaus were not found to lie at multiples of the quantum of conductance.

So far, even with carefully controlled conditions, it has not been possible to reproduce exactly the current evolution on breaking the contact. This is due to the intrinsic mechanism of plastic deformation: a stable atomic configuration is not reproduced in the elongating nanowires formed in successive experiments.
Fig. 3. Statistical study of 3000 , 6000,9000 and 12000 consecutive contact breakage conductance curves made using a constant speed of retraction $(8.9 \mu \mathrm{m} / \mathrm{s})$. A resistance of $490 \mathrm{~W}$ has been subtracted from the original data, this value being related to the average disorder in gold nanowires drawn at room temperature in air.

It has been argued by Olesen $e t$ al. [5] that a statistical study of a series of experiments must be made in order to prove the existence of quantized conductance during the contact between macroscopic wires.

Various criteria, essentially arbitrary, have been adopted in the past to select the conductance curves used to build histograms. To demonstrate the quantized nature of the conductance in nanowires we have performed a tour de force. A total of 12000 consecutive experiments provided a statistical sample some 100-times larger than those analyzed in previous studies, and selection criteria were not used. Fig. 3 shows the evolution of the conductance histogram, with its peaks at multiples of

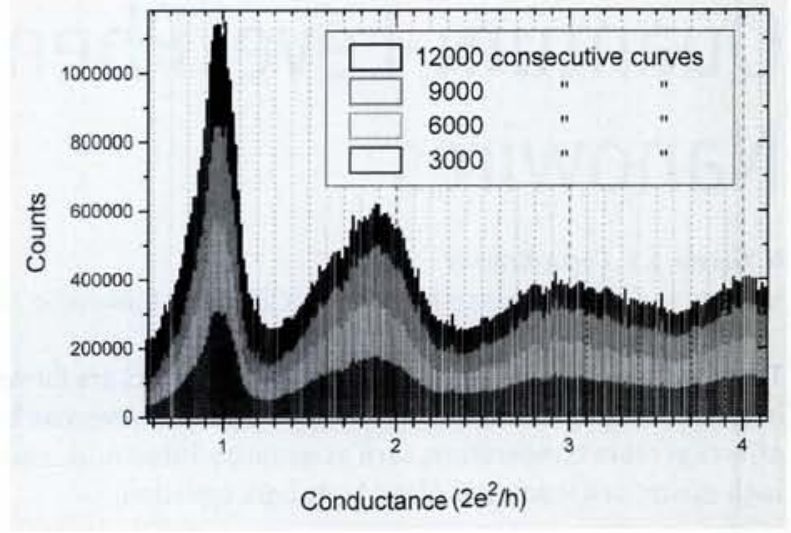

the quantum of conductance, for 3000 , 6000,9000 and 12000 consecutive conductance curves measured at room temperature using high-purity Au wires. We would like to stress that the uppermost histogram corresponds to data from the conductance curves measured on breaking 12000 consecutive times a contact between the same pair of Au wires. Moreover, conductance quantization observed in $\mathrm{Na}$ contacts appears to agree with calculated levels [8] for symmetric nanowires.

We believe that the first-order peak is better defined than the others because conductance is affected by disorderinduced backscattering in the nanowires. For instance, computer simulations indi

\section{Quantized Conductance}

To understand quantized conductance one needs to consider the situation depicted in figure (a) in which two reservoirs A and B, each with a Fermi energy $E_{\mathrm{f}}$ and the same inelastic mean free path $\lambda$ for the charge carrier, are connected by constriction such as a nanowire of length $L$ and width $W$. A current, $I$, will flow through the constriction when a potential difference $V$ is applied between $A$ and B. The current is ballistic if $\lambda>L$ and scattering due to disorder and surface roughness can be neglected. Under these conditions, the current $I_{j}$ transmitted by a channel $j$ can be written using the Landauer formalism [10] as

$$
I_{j}=e v_{j} \partial n_{j} / \partial E_{\mathrm{f}}(e V) T_{j}
$$

for a carrier velocity $v_{j}$, total transmission probability $T_{j}$ and electron mass $e$, The density of states for spin degeneration $\partial n_{f} / \partial E_{f}=$ $2 / h v_{j}$ where $h$ is thePlanck constant, so $I_{j}=$ $2 e^{2} T_{j} V / h$ and the quantum of conductance $G_{j}$ for spin degeneration for channel $j$ is given by $G_{j}=\left(\mathrm{d} I_{j} / \mathrm{d} V\right)_{V \rightarrow 0}=2 e^{2} T_{j} / h$ where the factor 2 arises from the spin degeneration. The total conductance $G$ is therefore

$$
G=\sum_{j} G_{j}=\left(2 e^{2} / h\right) \sum_{j} T_{j}
$$

In order to have steps of magnitude $2 e^{2} / h$ in
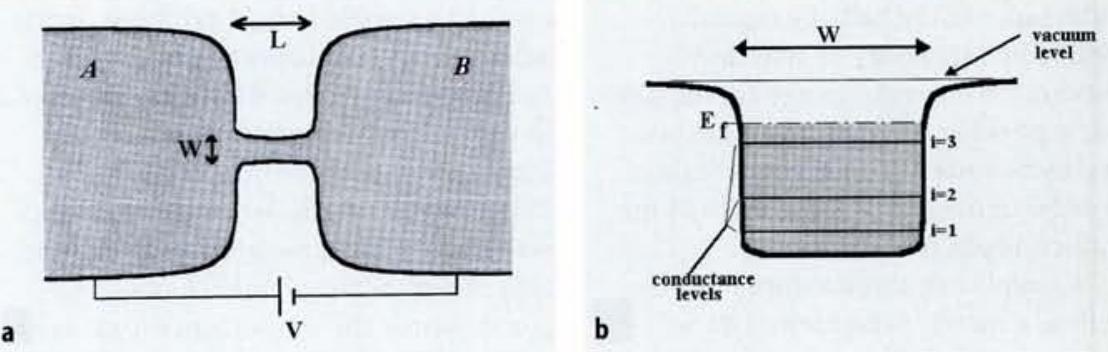

Quantized conduction in a nanowire. (a) A schematic drawing of the constriction (length $L$, width $W$ ) between two reservoirs $A$ and $B$ at a potential difference $V$. (b) The electronic states in the nanowire lie at different energies below the Fermi energy $E_{f}$ depending on the degeneracy $i$ of the level. (c) The conductance $G=2 e^{2} / h$ of the nanowire as a function of time as the wire necks down.

the conductance on breaking a nanocontact, $T_{j}$ should equal 1, i.e., elastic scattering at disorder or surface roughness should not arise because this will decrease the amplitude of the conductance jumps.

The channel $j$ denotes the number of transverse bound levels in the constriction, as in a waveguide where the power through the waveguide depends on the number of modes. The number of modes is $2 W / \lambda$ for a onedimensional constriction, and roughly speaking $(2 W / \lambda)^{2}$ for a two-dimensional constriction of the type formed in wire-breaking experiments, where the number will depend on the size and shape of the constriction.

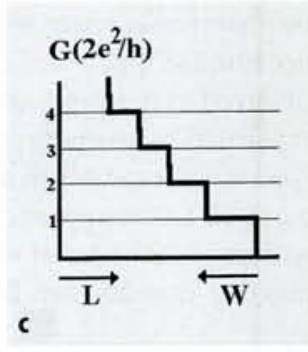

On changing the width of the constriction, every time a level exceeds the Fermi energy (equivalent to a new mode entering or leaving the conduction process), the conductance will increase by one or two quanta, depending on the degeneracy of the level. Accordingly, staircase $I-V$ conductance behaviour should be observed as a function of the conductor width $W$. 


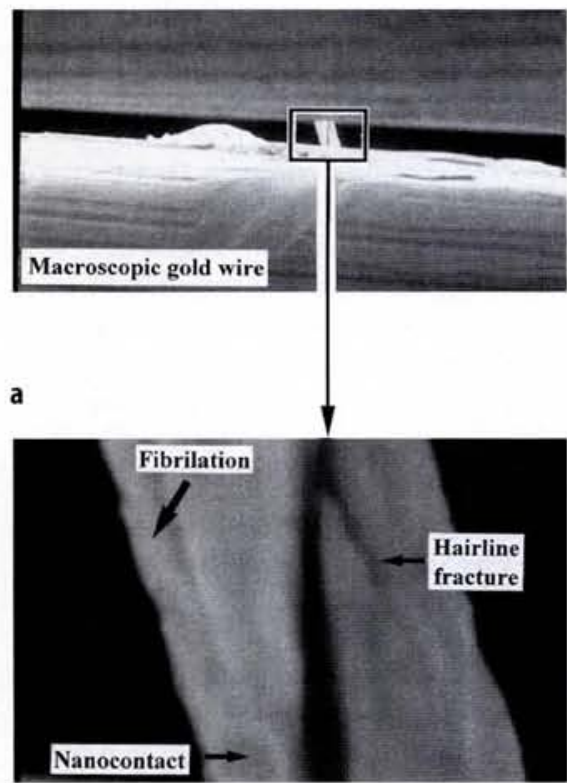

b

cate that for a constant degree of disorder in nanowires formed during successive contact separations, the higher-order peaks are more affected by backscattering than the first-order peak.

\section{Contact Area Structure}

Evidence for the formation of nanowires on separating two macroscopic wires has been obtained by twisting two wires together and then observing the contact area in a scanning electron microscope. The build up of torque fractures the contacts, leaving behind a texture characteristic of the kind of contact that was created.

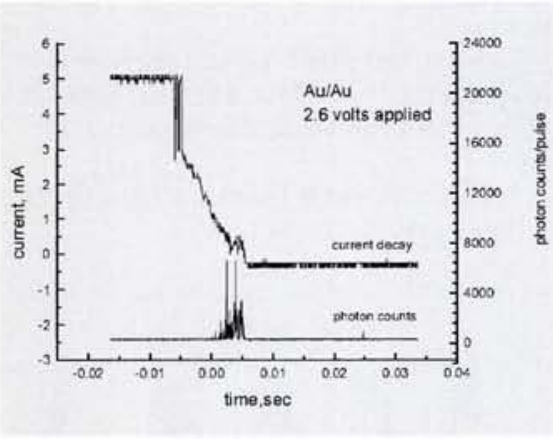

Fig. 5. A representative example of light emission from nanowires during the breaking of a contact between two macroscopic wires. The current (upper curve) observed during the contact breaking and photon emission (lower curve, in counts per pulse) are plotted as a function of time. Note that emission (involving a total of some tens of thousand of photons) took place within a time interval of $10 \mathrm{~ms}$ just before the contact broke. Experiments carried out within a collaboration using apparatus at both the University of Cambridge (M. Welland) and Madrid gave essentially the same results for different atmospheres (air, helium, oxygen and ultrahigh vacuum).

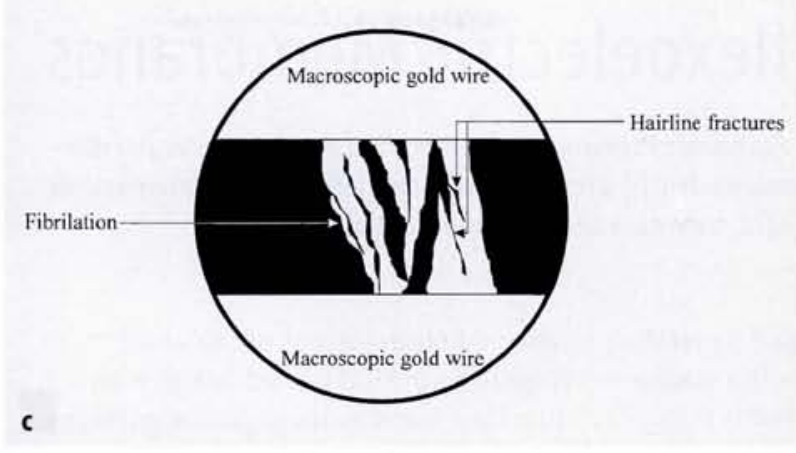

Fig. 4. Scanning electron micrograph (a) of a contact between high-purity macroscopic gold showing clear evidence for a bridge (magn.: $\left.\times 2.10^{3}\right)$. The enlargement $\left(\mathbf{b} ; \times 3.10^{4}\right)$ reveals the nanocontacts that make up the bridge. The bridge is divided into two threads $(\approx 400 \mathrm{~nm}$ width), each with small fibrillations $(\approx 50 \mathrm{~nm}$ width). Notice also the presence of small hairline fractures, which are also illustrated schematically in c.

Representative micrographs (Fig. 4) reveal small threads with diameters in the $50 \mathrm{~nm}$ range [9]. The microscope has a resolution of $6 \mathrm{~nm}$, but this level is difficult to reach because the thin wires have very low contrast. However, the texture observed clearly resembles metallic pizza cheese.

\section{Light Emission at Large Excitation}

Very recent experiments have demonstrated a novel phenomenon, namely light emission from the nanowires during the last stages of the contact, i.e., when the contact contains only a few conductance levels. The emission is observed just before the contact breaks at applied voltages above about $1 \mathrm{~V}$, and the number of photons emitted approaches some ten thousand per contact. Fig. 5 for an applied voltage of $2.5 \mathrm{~V}$ shows that the electric current (upper curve) passing through the contact lies within the $\mathrm{mA}$ range and is not quantized owing to non-linear effects at large excitations. Emission is not due to transitions between the conductance levels, which are still present, but to blackbody radiation with measured photons energies of 2-3 eV owing to the existence of very hot $(10000 \mathrm{~K})$ electrons in addition to cold phonons (atom vibrations are small since the nanowires are stable). These observations confirm that phenom- ena arising at the quantum level can be probed at room temperature using the fairly straightforward contact between macroscopic pieces of metal.

\section{Acknowledgments}

The authors wish to thank P. García-Mochales, P.A. Serena and L. Bitar, and are indebted to A. Correia who provided the SEM images used in Fig. 4 prior to their publication and to A. Gil and M. Sharonov for their results on light emission. The work was supported by the European Union (BRITE and ESPRIT) and Spain's DGCIT and CICYT agencies.

\section{References}

[1] N. García \& L. Escapa, Appl. Phys. Lett. 54 (1989) 1418; N. García, STM Workshop (ICTP, Trieste, 1987).

[2] J. Gimzewski, STM'87 Cónf. (Oxnard, CA, USA) 1987.

[3] B.J. van Wees et al., Phys. Rev. Lett. 60 (1988) 848; D.A. Wharam et al., Solid State Phys. 21 (1988) L209.

[4] J.I. Pascual et al., Phys. Rev. Lett. 71 (1993) 1852; J.I. Pascual et al., Science 267 (1995) 1793. [5] L. Olesen et al., Phys. Rev. Lett. 72 (1994) 2251; idem, Phys. Rev. Lett. 74 (1995) 2142; M. Brandbyge et al., Phys. Rev. B 52 (1995) 8499. [6] U. Landman et al., Science 248 (1990) 454. [7] J.L. Costa-Krämer, N. García, P. GarcíaMochales \& P.A. Serena, Surf. Sci. 342 (1995) L1144; Phys. Today (February 1996) 9.

[8] J.M. Krans et al., Nature 375 (1995) 767; J.A. Torres et al., Phys. Rev. B 49 (1994) 14721. [9] A. Correia, N. García (submitted). [10] R. Landauer, J. Phys.: Cond. Matter 1 (1989) 8099.

\section{Date: 15-17 October 1996}

Title: Physics and Application of Non-Crystalline Semiconductors in Optoelectronics: NATO-ARW

Venue: Kishinau, Republic of Moldova

$$
\text { Contact for information: }
$$

Prof. A.A. Andriesh, phone 00373-2-233587

Academy of Sciences bd. Stefan cel Mare n.1, Kishinau, Republic of Moldova

\section{A/Ab: August 20 $\bullet p p \cdot$ number of participants about 100} Language: English 